\title{
Multiple Trypanosoma infections are common amongst Glossina species in the new farming areas of Rufiji district, Tanzania
}

Imna I Malele $1^{*}$, Henry B Magwisha², Hamisi S Nyingilili', Kamilius A Mamiro², Elipidius J Rukambile², Joyce W Daffa ${ }^{3}$, Eugene A Lyaruu', Lupakisyo A Kapange', Gideon K Kasilagila', Nicodemus K Lwitiko", Halifa M Msami ${ }^{2}$ and Elikira N Kimbita ${ }^{5}$

\begin{abstract}
Background: Tsetse flies and trypanosomiasis are among several factors that constrain livestock development in Tanzania. Over the years Rufiji District was excluded from livestock production owing to tsetse fly infestation, however, a few years ago there was an influx of livestock following evictions aimed at conserving the Usangu wetlands.

Methods: A study was conducted to determine the efficiency of available traps for catching tsetse flies, Glossina species infesting the area, their infection rates and Trypanosoma species circulating in the area. Trapping was conducted during the semi dry season for a total of 30 days (ten days each month) during the onset of the dry season of May - July 2009. Harvested flies after every 24 hours were dissected and examined under a light microscope for trypanosome infections and whole fly DNA was extracted from 82 flies and analyzed for trypanosomes by polymerase chain reaction (PCR) using different sets of primers.

Results: The proportions of total tsetse catches per trap were in the following decreasing order S3 (33\%), H-Trap (27\%), Pyramidal (19\%), sticky panel (11\%) and biconical trap (10\%). Of the 1200 trapped flies, $75.6 \%$ were identified as Glossina pallidipes, $11.7 \%$ as G. brevipalpis, $9.6 \%$ as G. austeni and 3.0\% G. morsitans morsitans. Dissections revealed the overall infection rate of $6.6 \%$ (13/197). Whole DNA was extracted from 82 tsetse flies and the prevalence of trypanosomes circulating in the area in descending order was 92.7\% (76/82) for T. simiae; 70.7\% (58/ 82) for T. brucei types; 48.8\% (40/82) for the T. vivax types and 32.9\% (27/82) for the T. congolense types as determined by PCR. All trypanosome types were found in all tsetse species analysed except for the $T$. congolense types, which were absent in G. m. morsitans. None of the T. brucei positive samples contained human infective trypanosomes by SRA - PCR test

Conclusion: All tsetse species found in Rufiji are biologically important in the transmission of animal trypanosomiasis and the absence of T. congolense in G. m. morsitans could be a matter of chance only. Therefore, plans for control should consider all tsetse species.
\end{abstract}

Keywords: tsetse, Trypanosoma, Glossina, traps, attractants, livestock, trypanosomiasis, pastoralists, Tanzania

\footnotetext{
* Correspondence: malele2i@yahoo.com

'Tsetse \& Trypanosomiasis Research Institute (TTRI), Box 1026 Tanga,

Tanzania

Full list of author information is available at the end of the article
} 


\section{Background}

Tsetse flies and trypanosomiasis are a serious constraint to livestock sector development in sub Saharan Africa. The disease lowers productivity in livestock, reduces cattle density up to $70 \%$, sale of meat and milk by $50 \%$ and calving rates by $20 \%$ and calf mortality by $20 \%$ [1]. In Tanzania, tsetse-borne diseases, and in particular bovine trypanosomiasis, which is one of the two most important diseases that are responsible for reduced livestock productivity and together with tick-borne diseases they are responsible for $75 \%$ of the morbidities and mortalities in cattle [2].

Southern Tanzania is one of the areas in the country that are tsetse infested and keeping of livestock has been severely constrained by tsetse transmitted trypanosomiasis [3]. In early 2007, the country saw a rapid increase in the number of livestock settled in Rufiji district, of the Coastal Region of Tanzania, following the evacuation of livestock from Usangu and Ihefu areas which were declared conservation areas and are the key water sources for hydroelectric power generation [4]. Many pastoralists opted to settle in the Coastal Region, which has a low human population density hence ensures ample grazing land for their animals. Settlement of pastoralists and their animals in the district started a long time ago following pressures associated with land use in the northern circuit of the country [5]. However, animal populations in Rufiji district increased rapidly as a result of this eviction from the wetland sources from 20,000 livestock in $2005 / 2006$ to about 140,000 by mid $2008 / 2009$.

Settlement of these animals came without much preparation to avert the problem caused by tsetse and trypanosomiasis in the pasture areas. There was no baseline information on the situation of tsetse and trypanosomiasis; so despite the availability of plenty of grazing areas for animals, the pastoralists were confronted by a serious challenge of tsetse and trypanosomiasis, which has become a major stumbling block to livestock sector development in Rufiji. Records on tsetse and trypanosomiasis in southern Tanzania are very scant and the available records are by Connor and Halliwell [6] and MacLennan [3], who reported on bovine trypanosomiasis and tsetse distribution in Mtwara region respectively, an area bordering Mozambique. Prior to this study, there was no information regarding tsetse and trypanosomiasis in the Rufiji district.

This study was therefore conducted in order to investigate the magnitude of tsetse and trypanosomiasis distribution through investigation of tsetse species, densities, infection rates, trypanosome types circulating in the area in order to determine the magnitude of tsetse and trypanosomiasis ( $\mathrm{T} \& \mathrm{~T}$ ) for the purpose of planning control interventions against the vector and trypanosomiasis for improved livestock production in the District. The information gathered will contribute towards planning a control intervention against $\mathrm{T} \& \mathrm{~T}$ not only in Rufiji and the Coastal regions, but in southern Tanzania as a whole; against African Animal Trypanosomiasis (AAT) and Human African Trypanosomiasis (HAT) by bearing in mind that Rufiji is at the periphery of the southern silent foci of sleeping sickness, Nachingwea [7]. Control of $\mathrm{T} \& \mathrm{~T}$ will significantly improve agricultural activities and human health in the area.

\section{Materials and methods}

\section{Study site}

Rufiji district located at S-8.1022 latitude and $\mathrm{E}$ $38.3756^{\circ}$ longitude; is one of the districts in the Coastal Region and offers potential for agricultural and livestock production. The district covers 39.8 percent of the total $33,539 \mathrm{~km}^{2}$ of Coastal region area, which offers potential for mixed agriculture [8]. The average rainfall in this zone ranges from $900 \mathrm{~mm}$ - $1000 \mathrm{~mm}$ per year. Formerly, out of the total countrywide population of livestock, only $0.44 \%$ of the cattle and $1.7 \%$ of the sheep and goat populations were available in the area before the mass settlement in 2007 [8]. According to the 2002 national census, Rufiji had about $22.8 \%(202,001)$ of the total population of the Coastal region, which stood at 885,017 . The district is also endowed with 482,466 potential arable hectares; however, only $20.7 \%$ is utilized for crop production. Residents practice subsistence farming of crops like cassava, rice, maize, sweet potatoes and legumes, sorghum, cashew nuts and coconuts and fruits of various types especially oranges and mangoes. Small scale fishing is practiced in the Indian Ocean and Rufiji River [8]. The influx of pastoralists from the northern areas of the country is set to increase agro pastoral practices in the district.

\section{Entomological surveys Trap deployment}

For easy implementation of the study, the district was divided into three blocks (A, B and C) as follows:

Block A - covering Mkongo Division, Utete Ward Traps were deployed in Siasa Village, Utete West, situated on the western part of the district adjacent to the wildlife management area bordering Selous Game Reserve.

Block B - Kikale Division, Mtunda Ward Traps were deployed in Muyuyu village near Mtunda forest. This ward is situated on the northern part of Rufiji River.

Block C - Covering Muhoro Division, Chumbi Ward Traps were deployed in Kiwanga and Chumbi villages in the southern part of the district (Figure 1). 


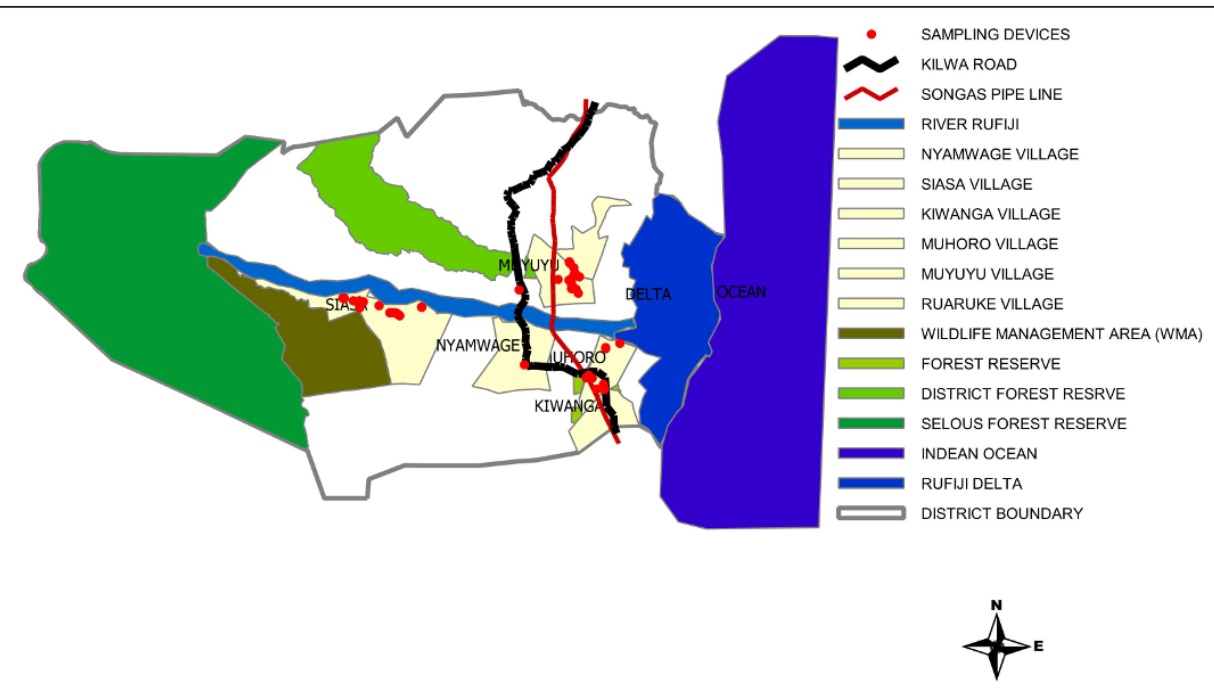

Figure 1 Tsetse surveyed areas in Rufiji District.

\section{Trapping of tsetse flies}

Information about the study area was obtained from the District Veterinary personnel before initiating the study. Having identified the tsetse infested areas, a total of 15 traps were deployed in selected points along transects in order to cover all different vegetations that were found in the three blocks. These included conventional stationary traps namely Biconical [9], Pyramidal [10], S3 [11], H-trap [12] and sticky panels [13]. In each block, five different traps were used and these were deployed at $10.00 \mathrm{am}$ every morning and emptied at the same time the next day. They were placed 150 - 200 metres apart depending on the vegetation cover of the area surveyed. All traps were baited with acetone, released from a $6 \mathrm{~mm}$ diameter hole in the top lid of a half litre plastic container. Study areas and trap locations were geo-referenced by a Global
Positioning System (GPS). The trapped flies were collected and sorted into respective sexes, species and numbers. Trapping was conducted during the semi dry season for a total of 30 days (ten days each month) during the onset of the dry season i.e. from May to July 2009.

Determination of tsetse species, infection rates and trypanosome types circulating in the area

Trapped tsetse were harvested after every $24 \mathrm{hrs}$, counted and sorted into respective species and sexes. The flies were then dissected and organs examined under a light microscope to determine the presence of trypanosomes. The flies were dissected in physiological buffered saline (PBS) using a stereo dissection microscope and target tissue (mouth parts, salivary gland and midguts) and fly carcasses were placed in $100 \%$ ethanol for storage at $-20^{\circ} \mathrm{C}$ and later DNA extraction. DNA from whole flies from 82

Table 1 Trypanosome species specific Primers used in this study

\begin{tabular}{|c|c|c|c|}
\hline Primer Code & Sequence & Base pairs (bp) & Reference \\
\hline $\mathrm{TV}$ & $\begin{array}{l}\text { 3-ACTCAAAATCGTGCACCTCG-5 } \\
\text { 5-CCCGGCAGGTTGGCCGCCATC-3 }\end{array}$ & 399 & {$[21]$} \\
\hline$\overline{\mathrm{TCK}}$ & $\begin{array}{l}\text { 5-GTG CCC AAA TाT GAA GTG AT-3 } \\
\text { 5-ACT CAA AAT CGT GCA CCT CG-3 }\end{array}$ & 294 & {$[22]$} \\
\hline$\overline{\mathrm{TCF}}$ & $\begin{array}{l}\text { 5-GGA CAC GCC AGA AGG TAC TT-3 } \\
\text { 5-GTT CTC GCA CCA AAT CCA AC-3 }\end{array}$ & 350 & {$[22]$} \\
\hline$\overline{\mathrm{TCS}}$ & $\begin{array}{l}\text { 5-CGA GAA CGG GCA CTा TGC GA-3 } \\
\text { 5-GGA CAA AGA AAT CCC GCA CA-3 }\end{array}$ & 316 & {$[22]$} \\
\hline $\mathrm{TBR}$ & $\begin{array}{l}\text { 5-CGAATGAATATTAAACAATGCGCAG-3 } \\
\text { 5-AGAACCATTATTAGCTTGTTGC-3 }\end{array}$ & 177 & {$[23]$} \\
\hline TSM & $\begin{array}{l}\text { 5-CCGGTCAAAAACGCATT-3 } \\
\text { 5-AGTCGCCCGGAGTCGAT-3 }\end{array}$ & 437 & {$[22]$} \\
\hline SRA A \& E & $\begin{array}{l}\text { A (5-GACAACAAGTACCTTGGCGC-3) } \\
\text { E (5-TACTGTTGTTGTACCGCCGC-3) }\end{array}$ & 460 & {$[24]$} \\
\hline Trypanosoma spp (ITS 1 CF \& ITS 1 BR) & $\begin{array}{l}\text { 5'-CCGGAAGTTCACCGATATTG-3' } \\
\text { 5'-TTGCTGCGTTCTTCAACGAA-3' }\end{array}$ & Species-specific sizes & {$[25]$} \\
\hline
\end{tabular}


Table 2 Tsetse species and apparent densities in the surveyed areas of Rufiji district

\begin{tabular}{|c|c|c|c|c|c|c|}
\hline Blocks & Division & Ward & Village & $\begin{array}{l}\text { Tsetse } \\
\text { species }\end{array}$ & Overall Total flies caught & $\begin{array}{l}\text { Overall Apparent density (Flies/trap/ } \\
\text { day) }\end{array}$ \\
\hline$A$ & Mkongo & Utete & Siasa & $\begin{array}{l}\text { G. pallidipes } \\
\text { G. brevipalpis } \\
\text { G. austeni } \\
\text { G. m. } \\
\text { morsitans }\end{array}$ & $\begin{array}{l}400 \text { ( } 154 \text { males, } 246 \\
\text { females) }\end{array}$ & 0.90 \\
\hline B & Kikale & Mtunda & Muyuyu & $\begin{array}{l}\text { G. pallidipes } \\
\text { G. brevipalpis } \\
\text { G. austeni }\end{array}$ & 99 (10 males, 89 females) & 0.22 \\
\hline$C$ & Muhoro & Chumbi & $\begin{array}{l}\text { Kiwanga near Kiwanga } \\
\text { forest. }\end{array}$ & $\begin{array}{l}\text { G. pallidipes } \\
\text { G. brevipalpis } \\
\text { G. austeni }\end{array}$ & $\begin{array}{l}700 \text { ( } 200 \text { males, } 500 \\
\text { females) }\end{array}$ & 1.60 \\
\hline
\end{tabular}

tsetse flies were also extracted using a Qiagen Kit for trypanosome characterization as previously described [14] showing that trypanosomes can be analysed from DNA extracted from whole flies without dissection. Amplification using Phusion Taq (FinnEnzyme) for different species-specific primers and test primers in a DNA thermal cycler (Applied Biosystem) in a final volume of $25 \mu \mathrm{l}$ was carried out following published protocols and amplification conditions as indicated in Table 1.

Internal transcribed spacer (ITS) amplification was carried out in $20 \mu \mathrm{l}$ reaction mixture containing $11.4 \mu \mathrm{l}$ distilled water, $4 \mu \mathrm{l}$ of $5 \times$ Phusion HF buffer (FinnEnzyme), $0.4 \mu \mathrm{l} 10 \mathrm{mM}$ dNTPs, $1 \mu \mathrm{l}$ for each Primer, 0.2 ul Phusion DNA polymerase and $2 \mu \mathrm{l}$ DNA template except for screening the presence of serum associate (SRA) gene, where $4 \mu \mathrm{l}$ of template was used. The polymerase chain reaction (PCR) condition involved an initial denaturation step at $98^{\circ} \mathrm{C}$ for 30 second, followed by 30 cycles of $98^{\circ} \mathrm{C}$ for $30 \mathrm{~s}, 60^{\circ} \mathrm{C}$ for $40 \mathrm{~s}, 72^{\circ} \mathrm{C}$ for $30 \mathrm{~s}$ with a final elongation step at $72^{\circ} \mathrm{C}$ for $5 \mathrm{~min} .5 \mu \mathrm{l}$ of PCR product was mixed with standard loading dye (Hyperladder) and electrophoresed in 1.5\% agarose stained with ethidium bromide $(5 \mu \mathrm{g} /$ $\mathrm{ml}$ ) and the product visualized and photographed under ultraviolet illumination. A positive control (with reference genomic DNA) and negative control (without DNA, only with distilled water) were included in each set of reactions.

\section{Results}

Entomology surveys: Tsetse composition and distribution A total of 1200 flies were trapped during this study and four tsetse species were identified as Glossina pallidipes, G. brevipalpis, G. m. morsitans and G. austeni. The proportional abundance of all trapped flies were $75.6 \%$ (908) for G. pallidipes, 11.7\% (140) for G. brevipalpis, 9.7\% (116) for G. austeni and 3.0\% (36) G. m. morsitans. Flies trapped in all blocks/villages sampled are indicated in Table 2.

\section{Tsetse fly response to traps}

$\mathrm{S} 3$ and $\mathrm{H}$ traps caught more flies than other traps (Figure 2). G. pallidipes was found in all traps used in this study, whereas G. m. morsitans was trapped by H-Trap and Sticky panels. G. austeni was trapped by S3, H-Trap and Sticky panels. In addition, G. brevipalpis were found trapped by $\mathrm{H}$ and Pyramidal traps.

\section{Infection rates in tsetse}

(i) Examination of dissected tsetse flies by microscopy

A total of 197 G. pallidipes were dissected and examined microscopically for trypanosomes and the overall infection rate ranged from $2.5 \%$ in midguts and $6.6 \%$ in proboscides and most of the infecting trypanosomes were $T$. vivax and $T$. congolense. The results are presented in Table 3.

\section{(ii) Molecular characterization of trypanosomes}

Results of DNA from 82 flies analyzed for trypanosomes by PCR are shown in Table 4. Initially, the intention was to determine trypanosome infection by ITS - PCR (Figure 3); however due to high prevalence of mixed infection it was decided to use species specific primers in order to identify the exact trypanosome species circulating in the area; also by bearing in mind that this was

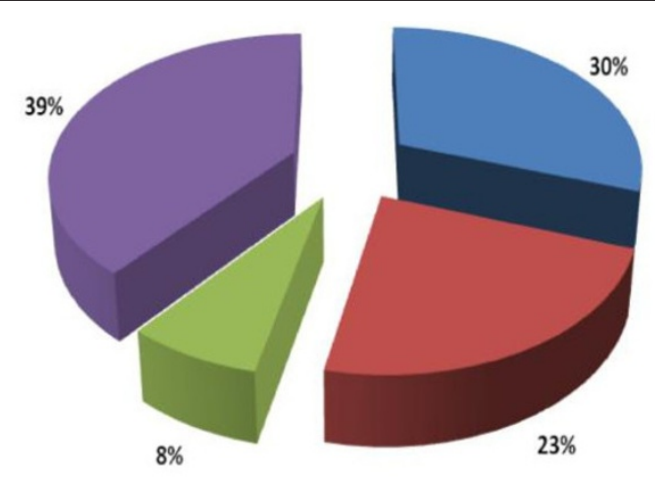

Figure 2 Proportion of trypanosomes types circulating in Rufiji. . Trypanosoma simiae - 39\%. T. brucei brucei - 30\%. T. vivax-8\%. T. congolense - 23\%. T. congolense includes T. congolense savannah, $\mathrm{T}$. congolense kilifi, and T. congolense forest. 
Table 3 Microscopic infection rates in tsetse

\begin{tabular}{llll}
\hline Tsetse species & Infected organ & Trypanosome spp/type & $\%$ infection \\
\hline G. pallidipes & proboscides & T. vivax & $13(6.6 \%)$ \\
\hline & midgut & T. brucei & $5(2.5 \%)$ \\
\hline & midgut & T. congolense & $5(2.5 \%)$ \\
\hline
\end{tabular}

the first time molecular characterization of trypanosomes circulating in tsetse from this area was carried out (Table $4 \&$ Table 5). None of the T. brucei positive samples contained human infective trypanosomes by SRA - PCR test. The proportions of trypanosome types circulating in the area are presented in Figure 4.

\section{The $T$. congolense genotypes}

The three T. congolense genotypes were found circulating in the area as indicated in Table 5.

\section{Pattern of Mixed infection of trypanosomes}

The pattern of mixed infection and occurrence was $13.1 \%(11 / 82)$ for single infection; 37.8 (31/82) for double; $35.4 \%$ (29/82) for triple infection. Those which occurred in a mixture of four together were 9.8\% (8/82). Only one (1.2\%) set of infection had a mixture of five different types of trypanosomes.

The proportion of analysed samples from each block was 19.5\% (16) for Utete (Block A); $24.4 \%$ (20) for block B (Muyuyu); 56.1\% (46) for block C (Kiwanga and Muhoro).

\section{Discussion and conclusion}

The study found that the area was infested by four species of tsetse, namely G. pallidipes, G. m. morsitans, G. brevipalpis and G. austeni. Individual tsetse species responded differently to the traps used in the study. G. pallidipes were found in all traps namely Biconical, Pyramidal, S3, H-trap and Sticky panel. G. m. morsitans were found in H-trap and Sticky panel; G. austeni were found in S3, H-trap and sticky panel whereas G. brevipalpis were also found in Pyramidal and H-trap. The apparent density obtained was not very high compared to flies seen visiting most traps. This could be due to the reluctance of most Morsitans tsetse flies to enter into stationary traps. Observation made by
Shaw et al. [15] on G. pallidipes and G. morsitans indicated that only $47-30 \%$ of tsetse approaching a trap landed on it or entered it, which means that about $53-70 \%$ of tsetse visiting the trap did not contact it. This is the phenomenon that was also noticed in Rufiji. Flies would land on traps without entering the retaining cage.

About five species of trypanosomes, the causative parasites of AAT in livestock were found circulating in tsetse. We used species specific primers in order to obtain the exact types of species found in the area. However, we did not use the $T$. godfreyi primers, or the T. vivax specific primers for $T$. vivax western types, which could have affected the $T$. vivax prevalence in the area and the total number of trypanosomes circulating in the area. So far tsetse flies found in Rufiji District are infected by $T$. vivax, T. simiae, T. brucei types and the three genotypes of $T$. congolense ( $T$. congolense savannah, T. congolense forest and T. congolense kilifi). $T$. simiae included the $T$. simiae Tsavo and T. congolense Tsavo [16].

The $T$. congolense types were pooled together to give the total congolense types in the area (Table 4); however, when analysed individually, G. m. morsitans were not infected by the $T$. congolense parasite. Trypanosoma congolense kilifi was found in G. pallidipes (8.5\%), G. brevipalpis $(2.4 \%)$ and G. austeni (4.9\%). Trypanosoma congolense forest was only found in G. pallidipes (1.2\%). Trypanosoma congolense savannah was found in all tsetse species analysed except in G. m. morsitans. None of the T. brucei positive samples were SRA - PCR positive. It was important to have a confirmatory test of the infective status of T. brucei positive samples because Rufiji is at the periphery of the southern silent sleeping sickness foci (Nachingwea). Also with movements of animals from northern and western parts of Tanzania through tsetse infested HAT active foci; the likelihood of human infective trypanosomes being

Table 4 Trypanosomes infection rates per tsetse species

\begin{tabular}{|c|c|c|c|c|}
\hline Tsetse spp & $\mathrm{Tb}$ & $\mathrm{TC}^{*}$ & Tv & Tsm \\
\hline G. pallidipes & 45/82 (54.9\%) & 19/82 (23.2\%) & 26/82 (31.7\%) & 58/82 (70.7\%) \\
\hline G.m. morsitans & $3 / 82(3.7 \%)$ & 0 & $3 / 82(3.7 \%)$ & $4 / 82(4.9 \%)$ \\
\hline G. brevipalpis & $4 / 82(4.9 \%)$ & $3 / 82(3.7 \%)$ & $4 / 82(4.9 \%)$ & $7 / 82$ (8.54\%) \\
\hline G. austeni & $6 / 82(7.3 \%)$ & $5 / 82(6.1 \%)$ & $7 / 82(8.54 \%)$ & $7 / 82$ (8.54\%) \\
\hline Overall & $58 / 82(70.73 \%)$ & $27 / 82$ (32.93\%) & $40 / 82$ (48.78\%) & $76 / 82(92.7 \%)$ \\
\hline
\end{tabular}

* Includes the total genotypes of $T$. congolense. $T b=T$. brucei; $T C=T$. congolense; $T v=T$. vivax; and $T s m=T$. simiae $T$ savo and $T$. congolense $T$ savo 


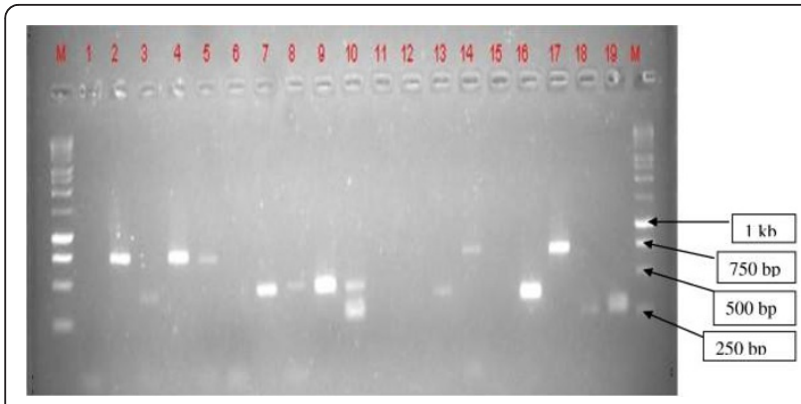

Figure 3 Trypanosome DNA products by ITS - PCR from Rufiji tsetse samples. Numbers 4, 5, 14, and 17 are $T$. congolense savannah $\sim 700 \mathrm{bp}$. Numbers 3,7,8,9,10,13,16 are T. simiae $\sim 400 \mathrm{bp}$. Numbers 10 and 19 are T. savannah Tsavo 370 bp. Number 18 is T. vivax 250 bp. Number No. 2 is positive control for T. congolense savannah. Number 1 is negative control. $M=1 \mathrm{~kb}$ DNA ladder.

introduced in Rufiji cannot be ignored. In areas like Uganda, livestock movements have been behind the spread of HAT in the country [17].

The results have indicated the importance of G. austeni and G. brevipalpis in the epidemiology of animal trypanosomiasis through harboring animal infective parasites. It was also interesting to note the presence of T. brucei and T. vivax infections in G. brevipalpis as well as G. austeni. Available documented reports tend to link G. austeni and G. brevipalpis with Suidae related trypanosomes which are mainly $T$. simiae due to its feeding preferences of warthogs and hippopotamus [18], respectively. However, in this study, the two tsetse species (G. brevipalpis and G. austeni) were found to be equally infected with all trypanosome types circulating in the area more or less like the $G$. pallidipes and G. m. morsitans species which are known to play a major role in the epidemiology of AAT. The high prevalence of $T$. simiae in the area gives the indication that pig production in Rufiji District could be adversely affected or rather impossible.

\section{Pattern of trypanosome infections}

The common single infections were the $T$. simiae with total occurrence of 8, six in G. pallidipes and one each in G. brevipalpis and G. m. morsitans and none in G.

Table 5 The prevalence of T.congolense genotypes per tsetse species

\begin{tabular}{llll}
\hline Tsetse spp & Tck & Tcf & Tcs \\
\hline G. pallidipes & $7 / 82(8.5 \%)$ & $1 / 82(1.22 \%)$ & $11 / 82(13.4 \%)$ \\
\hline G. m. morsitans & $0 / 82$ & $0 / 82$ & $0 / 82$ \\
\hline G. brevipalpis & $2 / 82(2.4 \%)$ & $0 / 82$ & $1 / 82(1.22 \%)$ \\
\hline G. austeni & $4 / 82(4.9 \%)$ & $0 / 82$ & $1 / 82(1.22 \%)$ \\
\hline Overall & $\mathbf{1 3 / 8 2 ( 1 5 . 8 5 \% )}$ & $\mathbf{1 / 8 2}(\mathbf{1 . 2 2} \%)$ & $\mathbf{1 3 / 8 2 ( 1 5 . 8 5 \% )}$
\end{tabular}

NB: $T c k=T$. congolense kilifi; $T c f=T$. congolense forest; and $T c s=T$. congolense savannah

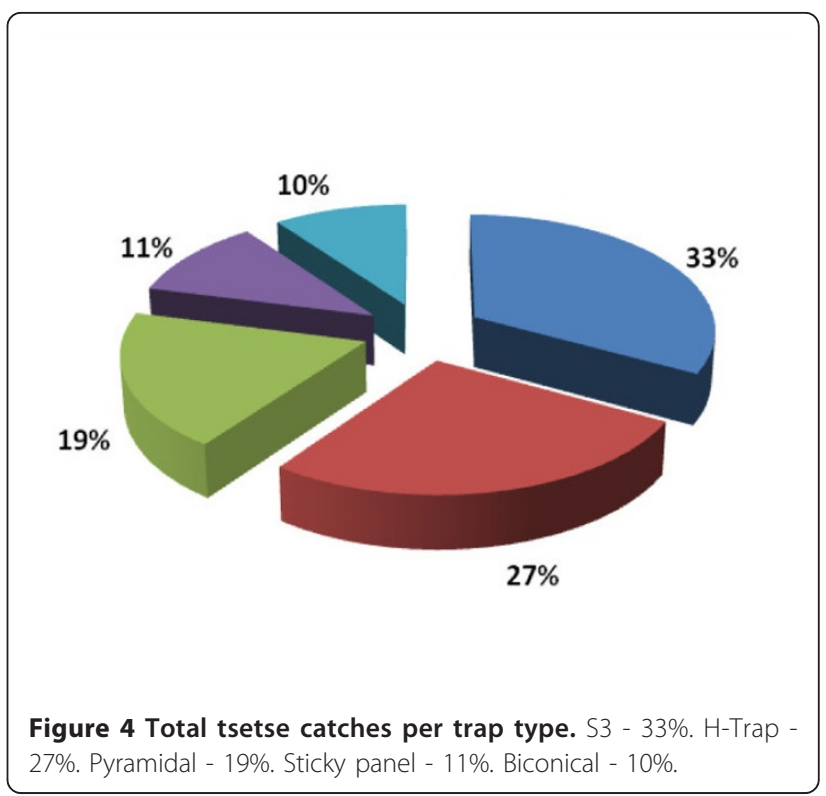

austeni. The least common single infection pattern was the $T$. vivax. The $T$. congolense forest ( $\mathrm{Tcf}$ ) was found once in G. pallidipes only and occurred in a mixture of five infections $(\mathrm{Tb}+\mathrm{Tcf}+\mathrm{Tcs}+\mathrm{Tv}+\mathrm{Tsm})$. The common pattern of triple infection was $\mathrm{Tb}+\mathrm{Tv}+\mathrm{Tsm}$ with a total 19 scores, highest in G. pallidipes (14/19), followed in G. m. morsitans (3/19) and one each in G. austeni and G. brevipalpis. The common pattern of double infections was for $\mathrm{Tb}$ and Tsm with a total of 22, highest in G. pallidipes (20/22); one each in G. brevipalpis and G. austeni. Other double infections were for Tv and Tsm with total occurrences of 6, three in G. pallidipes; 2 in G. brevipalpis and 1 in G. austeni. Other patterns were of $\mathrm{Tb}+\mathrm{Tv}+\mathrm{Tcs}$ recorded in G. austeni only; whereas the infection of $\mathrm{Tb}+\mathrm{Tck}+\mathrm{T} \mathrm{cs}+\mathrm{Tsm}$ was recorded in $G$. brevipalpis only. The occurrence of four different infections of trypanosome in G. brevipalpis confirms earlier findings of Namabolo et al. [19], on the importance and role of G. brevipalpis and G. austeni in the epidemiology of AAT in tsetse infested areas in South Africa. Lastly there were three scores of $\mathrm{Tb}+\mathrm{Tck}+\mathrm{Tv}+\mathrm{Tsm}$ all recorded in G. austeni only (Table 6).

In this study we recorded the presence of the three distinct subspecies of $T$. congolense, which are known to have various severity/virulence in cattle, which is an indication of how important is AAT in the area. The western part of the district (Utete) borders the Selous game reserve, which could be a source of a cocktail of trypanosomes in tsetse as a result of close interactions between livestock and wild animals in the area. It is possible that tsetse flies in this area obtain blood meals from both wild and domestic animals, and this would increase the trypanosomes types found in the vector. 
Table 6 Trypanosome mixed infection patterns and their occurrence in tsetse

\begin{tabular}{|c|c|c|c|c|c|c|c|}
\hline $\begin{array}{l}\text { Number of trypanosome } \\
\text { species }\end{array}$ & $\begin{array}{l}\text { Pattern of trypanosome } \\
\text { infection }\end{array}$ & $\begin{array}{l}\text { G. } \\
\text { austeni }\end{array}$ & $\begin{array}{l}\text { G. } \\
\text { brevipalpis }\end{array}$ & $\begin{array}{l}\text { G. } m \text {. } \\
\text { morsitans }\end{array}$ & $\begin{array}{l}\text { G. } \\
\text { pallidipes }\end{array}$ & Total & $\begin{array}{l}\% \\
\text { prevalence }\end{array}$ \\
\hline 2 & $T v+T s m$ & 1 & 2 & & 3 & 6 & 7.32 \\
\hline 5 & $T b+T c f+T c s+T v+T s m$ & & & & 1 & 1 & 1.22 \\
\hline 4 & $T v+T c k+T c s+T s m$ & & & & 1 & 1 & 1.22 \\
\hline 3 & $T b+T c s+T s m$ & & & & 3 & 3 & 3.66 \\
\hline 2 & $\mathrm{Tck}+\mathrm{Tsm}$ & & & & 3 & 3 & 3.66 \\
\hline 4 & $\mathrm{~Tb}+\mathrm{T} v+\mathrm{Tcs}+\mathrm{Tsm}$ & & & & 2 & 2 & 2.44 \\
\hline 3 & $\mathrm{~Tb}+\mathrm{T} c k+\mathrm{Tsm}$ & & & & 2 & 2 & 2.44 \\
\hline 3 & $T v+T c s+T s m$ & & & & 2 & 2 & 2.44 \\
\hline 3 & $\mathrm{~T} c k+\mathrm{T} c s+\mathrm{Tsm}$ & & & & 1 & 1 & 1.22 \\
\hline 3 & $T b+T v+T s m$ & 1 & 1 & 3 & 14 & 19 & 23.17 \\
\hline 3 & $T c k+T v+T s m$ & 1 & & & 1 & 1 & 1.22 \\
\hline 2 & $\mathrm{~Tb}+\mathrm{Tsm}$ & 1 & 1 & & 20 & 22 & 26.83 \\
\hline 1 & Tsm & & 1 & 1 & 6 & 8 & 9.76 \\
\hline 1 & $\mathrm{~Tb}$ & & & & 4 & 4 & 4.88 \\
\hline 1 & TV & & & & 1 & 1 & 1.22 \\
\hline 3 & $\mathrm{~Tb}+\mathrm{T} v+\mathrm{Tcs}$ & 1 & & & & 1 & 1.22 \\
\hline 4 & $T b+T c k+T c s+T s m$ & & 1 & & & 1 & 1.22 \\
\hline \multirow[t]{2}{*}{4} & $\mathrm{~Tb}+\mathrm{T} c k+\mathrm{T} v+\mathrm{Tsm}$ & 3 & 1 & & & 4 & 4.88 \\
\hline & & 8 & 7 & 4 & 64 & 82 & 100.02 \\
\hline
\end{tabular}

$T b-T$. brucei; Tcs - T. congolense savannah; Tcf - T. congolense forest; Tck - T. congolense kilifi; Tv - T. vivax; Tsm - T. simiae Tsavo and T. congolense Tsavo

Mixed infections of trypanosomes seem to be a usual phenomenon in southern Tanzania. Connor \& Halliwell [6] reported 93 positive cattle with trypanosome of which $56 \%$ were infected by $T$. congolense; $17 \%$ by $T$. vivax and $2.2 \%$ with $T$. brucei. Five animals had mixed infections of the three groups of trypanosomes ( $\mathrm{Tc}, \mathrm{Tb} \& \mathrm{Tv})$. This points towards the severity of AAT in cattle in southern Tanzania and this exacerbated animal losses incurred by pastoralists when they moved to southern Tanzania [4] such that deliberate efforts need to be in place to control tsetse in order to break the transmission cycle to domestic animals and thus improve livestock production and productivity in the area.

AAT risk is usually linked to the density of the vector and the trypanosome infection rates and from this area both parameters exists [20] i.e. tsetse and high trypanosome infection rates. Each tsetse species found in the area is significantly important due to the fact that they were all more or less found infected by all types of trypanosomes circulating in the area. T. vivax and T. congolense are regarded as major pathogens of cattle and other ruminants, while T. simiae causes high mortality in domestic pigs. The trypanosome types of $T$. vivax, T. brucei and T. simiae were found in all four tsetse species found in the area; on the other hand T. congolense types were not found in $G$. $m$. morsitans. This is an indication that the dominance of $G$. pallidipes should not exclude other tsetse species when planning for tsetse control strategies. Kiwanga village which is near Kiwanga forest (Block C) was relatively undisturbed when we sampled, however, pastoralists had started settling in search of pasture for their animals and this could explain the relatively high density of flies compared to other blocks. Block B was affected by the settlement of pastoralists, as by the time of sampling much tree felling was underway to clear areas for farming and other daily related activities; whereas in block A (Utete) we were able to trap flies in the first ten days of May. In June the area was burnt, hence we couldn't trap flies despite the fact that the area is near the Selous game reserve where the fly density is high. The different types of trypanosomes found in tsetse directly implicates tsetse in the transmission of AAT in livestock; and this is the first study to show the role of tsetse species in the transmission of AAT in southern Tanzania.

\section{Acknowledgements}

The Government of the United Republic of Tanzania through the Zonal Agricultural Research and Development Fund (ZARDEF), Eastern Zone for funding (L/01/15 grant), TTRI \& CVL Directors for logistic support.

\section{Author details}

${ }^{1}$ Tsetse \& Trypanosomiasis Research Institute (TTRI), Box 1026 Tanga, Tanzania. ${ }^{2}$ Central Veterinary Laboratory (CVL), Box 9254 Dar Es Salaam, Tanzania. ${ }^{3}$ Ministry of Livestock Development \& Fisheries (MLDF), Box 9152, Dar Es Salaam, Tanzania. ${ }^{4}$ District Veterinary Office, Rufiji, Tanzania. ${ }^{5}$ Sokoine University of Agriculture (SUA), Box 3019, Morogoro, Tanzania. 


\section{Authors' contributions}

IIM, HBM, KAM, EJR, HMM and ENK conceived the study, designed and coordinated the study; IIM, HBM, ENK performed data analysis and drafted the manuscript. KAM, JWD, EAL, GKK, LAK and NKL participated in the field work. HSN, EAL, IIM and LAK carried out the laboratory analysis on collected samples. EAL, HSN drew the map of the sampling sites. All authors read and approved the final version of the manuscript.

\section{Competing interests}

The authors declare that they have no competing interests.

Received: 18 August 2011 Accepted: 17 November 2011 Published: 17 November 2011

\section{References}

1. Swallow B: Impacts of Trypanosomosis on African Agriculture. PAAT Technical and Scientific Series 2 FAO, Rome; 2000, 52.

2. Jahnke HE, Tacher G, Keil P, Rojat D: Livestock production in tropical Africa, with special reference to the tsetse-affected zone. Proceedings of a Meeting of the African Trypanotolerant Livestock Network 1988, 3-21, Nairobi, International Livestock Centre for Africa and the International Laboratory for Research on Animal Diseases.

3. MacLennan KJR: Mtwara/Lindi Regional Integrated Development Programme, Tanzania. A review of Glossina distribution and trypanosomiasis. LRDC Report TANZA-067/80, ODA, U.K; 1980

4. Ngailo JA: Assessing the effects of eviction on household food security of livestock keepers from the Usangu wetlands in SW Tanzania. Livestock Research for Rural Development 2011, 23(3):2011.

5. Malele II, Nyingilili $H$, Msangi A: Factors defining the distribution limit of tsetse infestation and the implication for livestock sector in Tanzania. African J Agric Res 2011, 6(10):2341-2347.

6. Connor RJ, Halliwell RW: Bovine trypanosomiasis in southern Tanzania: parasitological and serological survey of prevalence. Trop Anim Health Prod 1987, 19(3):165-72.

7. Leak SGA: Tsetse Biology and Ecology. Their Role in the Epidemiology and Control of Trypanosomosis. CAB International, Oxford; 1999, 98-100.

8. Tanzania Coast Region Socio - Economic Profile. 2007 [http://www. tanzania.go.tz/regions/COAST.pdf].

9. Challier A, Laveissiere C: Un nouveau piege pour la capture des glossines (Glossina: Diptera, Muscidae): description et essais sur le terrain. Cahiers ORSTOM, Series Entomologie Medical Parasitologie 1973, 11:251-262.

10. Gouteux JP, Lancien J: The pyramidal trap for collecting and controlling tsetse flies (Diptera: Glossinidae). Comparative trials and description of new collecting technics. Trop Med Parasitol 1986, 37(1):61-6.

11. Ndegwa PN, Mihok S: Development of odour-baited traps for Glossina swynnertoni (Diptera: Glossinidae). Bull Ent Res 1999, 89:255-261.

12. Kappmeier K: A newly developed odour-baited " $\mathrm{H}$ trap" for the live collection of Glossina brevipalpis and Glossina austeni (Diptera: Glossinidae) in South Africa. Onderstepoort J Vet Res 2000, 67(1):15-26.

13. Vreysen MJB, Saleh KM, Zhu Z-R, Suleiman FW: Responses of Glossina austeni to sticky panels and odours. Med Vet Entomol 2000, 14(3):283-289.

14. Filipa Ferreira, Jorge Cano, Andreia Furtado, Nicolas Mabale Ndong, Pedro Asumu Ndong, Agustin Benito, Sónia Lima Centeno, et al: An alternative approach to detect trypanosoma in Glossina (Diptera, Glossinidae) without dissection. J Infect Developing Countries 2008, 2(1):63-67.

15. Shaw A, Torr S, Waiswa C, Robinson T: Comparative Costings of Alternatives for Dealing with Tsetse: Estimates for Uganda. Rome: FAO; 2007.

16. Gibson WC, Stevens JR, Mwendia MT, Makumi JN, Ngotho JM, Ndung'u JM: Unravelling the phylogenetic relationships of African trypanosomes of suids. Parasitol 2001, 122:625-631.

17. Batchelor NA, Atkinson PM, Gething PW, Picozzi K, Fèvre EM, Kakembo ASL, Welburn SC: Spatial Predictions of Rhodesian Human African Trypanosomiasis (Sleeping Sickness) Prevalence in Kaberamaido and Dokolo, Two Newly Affected Districts of Uganda. PLoS Negl Trop Dis 2009, 3(12):e563.

18. Clausen PH, Adeyemi H, Bauer I, Breloeer B, Salchow F, Staak C: Host preferences of tsetse (Diptera: Glossinidae) based on blood meal identifications. Med Vet Entomol 1998, 12:169-180.
19. Namabolo MV, Ntantiso L, Latif A, Majiwa PAO: Natural infection of cattle and tsetse flies in South Africa with two genotypic groups of Trypanosoma congolense. Parasitol 2009, 136(4):425-431.

20. Salim B, Bakheit MA, Salih ES, Kamau J, Nakamura I, Nakao R, Sugimoto C: An outbreak of bovine trypanosomiasis in the Blue Nile State, Sudan. Parasit Vectors 2011, 4:74.

21. Morlais I, Ravel S, Grebaut P, Dumas V, Cuny G: New molecular marker for Trypanosoma (Duttonella) vivax identification. Act Trop 2001, 80:207-213.

22. Masiga DK, Smyth AJ, Hayes P, Bromidge TJ, Gibson WC: Sensitive detection of trypanosomes in tsetse flies by DNA amplification. Int J Parasitol 1992, 64:207-218.

23. Moser DR, Cook GA, Ochs DE, Bailey CP, McKane MR, Donelson JE: Detection of Trypanosoma congolense and Trypanosoma brucei subspecies by DNA amplification using the polymerase chain reaction. Parasitol 1989, 99:57-66.

24. Gibson W, Backhouse T, Griffiths A: The human serum-resistance associated gene is ubiquitous and conserved in Trypanosoma brucei rhodesiense throughout East Africa. Infect Genet Evol 2002, 1:207-214.

25. Njiru ZK, Constantine CC, Guya S, Crowther J, Kiragu JM, Thompson RCA, Dávila AMR: The use of ITS1 rDNA PCR in detecting pathogenic African trypanosomes. Parasitol Res 2005, 95:186-192.

doi:10.1186/1756-3305-4-217

Cite this article as: Malele et al: Multiple Trypanosoma infections are common amongst Glossina species in the new farming areas of Rufiji district, Tanzania. Parasites \& Vectors 2011 4:217.

\section{Submit your next manuscript to BioMed Central and take full advantage of:}

- Convenient online submission

- Thorough peer review

- No space constraints or color figure charges

- Immediate publication on acceptance

- Inclusion in PubMed, CAS, Scopus and Google Scholar

- Research which is freely available for redistribution

Submit your manuscript at www.biomedcentral.com/submit
Ciomed Central 